\title{
Optimal insurance contracts without the non-negativity constraint on indemnities: revisited
}

\author{
Michael Breuer
}

Received: 8 March 2005 / Revised: 19 October 2005

(C) Springer Science + Business Media, LLC 2006

\begin{abstract}
In the literature on optimal indemnity schedules, indemnities are usually restricted to be non-negative. Keeler [1974] and Gollier [1987] show that this constraint might well bind: insured could get higher expected utility if insurance contracts would allow payments from the insured to the insurer at some losses. This paper extends Gollier's findings by allowing for negative indemnity payments for a broader class of insurers' cost functions and argues that the indemnity schedule derived here is more appropriate for practical applications (e.g. in health insurance).
\end{abstract}

Keywords Optimal insurance $\cdot$ Indemnity $\cdot$ Deductible $\cdot$ Co-insurance

JEL Classification D80 - D81 - D89

\section{Introduction}

A standard exercise in the literature on optimal indemnity schedules is to restrict the set of feasible indemnity schedules. ${ }^{1}$ A common restriction, which looks most sensible, is that indemnities have to be non-negative. However, Keeler [1974] was the first to develop the idea that insurance may work more efficiently if payments in and out depend on each insured's loss state, rather than prescribing a fixed premium. Gollier [1987] proves this result mathematically for the case of a risk neutral insurer. He shows that the non-negativity constraint can be binding for some loss distributions (e.g. for chronically ill persons in health insurance). Gollier also derives an optimal indemnity schedule, which is piecewise linear: For losses between zero and a nonnegative

\footnotetext{
M. Breuer $(\bowtie)$

Socioeconomic Institute, University of Zurich, Hottingerstr 10,

8032 Zurich, Switzerland

e-mail: mbreuer@soi.unizh.ch

${ }^{1}$ See for example Mossin [1968], Gould [1969], Arrow [1971], Moffet [1977], Raviv [1979], Drèze [1981], Schlesinger [1981], Gollier and Schlesinger [1996] and Spaeter and Roger [1997].
} 
loss $x_{-}$indemnities are negative with full marginal indemnity. Beyond a nonnegative deductible $x_{+}$indemnities are positive with full marginal indemnity. For all losses between $x_{-}$and $x_{+}$indemnities are zero.

This paper strongly supports Gollier's basic idea but argues that some of his specific results depend heavily on the insurer's cost function imposed. By allowing for a more flexible cost function it is possible to derive indemnity schedules that are more appropriate for practical applications, e.g. in health insurance.

\section{The model}

\subsection{Assumptions}

Let risk averse individuals have utility function $U(A), U^{\prime}(A)>0, U^{\prime \prime}(A)<0$, with $A$ representing their net wealth. The risk neutral insurer is supposed to recover cost but to make zero expected profit. Therefore, premiums $(P)$ are equal to indemnities paid plus administrative costs $C(|I(x)|)$ that also emerge when indemnities are negative:

$$
P=\int_{0}^{L}(I(x)+C(|J(x)|)) f(x) d x,
$$

with $f(x)$ representing the density function of losses. We impose a maximal loss of $L$. In order to allow for increasing marginal costs, $C^{\prime}(|I(x)|) \geq 0$ and $C^{\prime \prime}(|I(x)|) \geq 0$.

Although the simplified insurer's cost function employed in (1) does not account explicitly for verification costs and shows no fixed costs per claim, it is a plausible specification for a range of applications. Consider e.g. a contract between a health insurer and a general practitioner in a gatekeeper model, where the patient has to pay the doctor. Payments between the patient and the insurer are based on information about the utilization of the doctor's services, which is shared between the doctor and the insurer. In such an arrangement, the insurer is not involved at all if indemnity is zero. Insurer's costs only evolve if indemnities are nonzero and may well rise with positive indemnity (due to verification and administration) and the absolute amount of negative indemnity (due to enforcement costs and administration).

In contrast, Gollier assumes insurer's costs to be given by $C(E(|I(x)|))$. This cost function is adequate if the insurer has to bear costs which depend exclusively on the average amount of losses (e.g. personnel, IT). It is, however, more realistic to allow costs to depend on the actual amount of losses, and hence use the specification in the premium function (1). Furthermore, observe that the cost function employed in (1) is compatible with Gollier's cost function if marginal costs are constant $\left(C^{\prime \prime}=0\right)$. Finally, note that if $C$ is concave, Gollier overestimates insurance costs.

Let $w$ denote individuals' exogenous wealth. The optimization problem then is to maximize insured's expected utility from net wealth $A(x)=w-P-x+I(x)$ :

$$
\int_{0}^{L} U(w-P-x+I(x)) f(x) d x
$$


subject to (1). ${ }^{2}$

\subsection{The optimal indemnity schedule}

In order to solve this problem, this paper adopts the optimal-control approach from Raviv (1979). In line with Raviv, the first step is to introduce the following state variable:

$$
\left.\Gamma(\hat{x})=-\int_{0}^{\hat{x}}(I(x))+C(|I(x)|)\right) f(x) d x
$$

The initial condition is $\Gamma(0)=0$. The terminal condition reads as $\Gamma(L)=-P$, which corresponds to a zero-profit constraint for the insurer. The corresponding Hamiltonian is

$$
H=U(w-P-x+I(x)) f(x)-\lambda(x)(I(x)+C(\mid I(x \mid)) f(x)
$$

The general properties of the optimal schedule can be derived by differentiating (4) w.r.t. $I(x)$. The FOC (after eliminating $\lambda$ and rearranging terms) reads:

$$
\left.\frac{U^{\prime}(w-P-x+I(x))}{\left(I-C^{\prime}(-I(x))\right)}\right|_{I(x)<0}=\left.\frac{U^{\prime}(w-P-x+I(x))}{\left(1+C^{\prime}(I(x))\right)}\right|_{I(x)>0} .^{3}
$$

As $I(x) \rightarrow 0,(5)$ might be rewritten as

$$
\frac{U^{\prime}\left(w-P-x_{-}\right)}{\left(1-C^{\prime}(0)\right)}=\frac{U^{\prime}\left(w-P-x_{+}\right)}{\left(1+C^{\prime}(0)\right)} .
$$

According to (6) $x_{-}=x_{+}$for $C^{\prime}(0)=0$. However, for positive marginal costs at $I(x)=0$ it must be true that $x_{-}<x_{+}$. No positive lower bound $x_{-}$can be determined if $C^{\prime}(0) \geq 1$

In a manner similar to Raviv, one can also derive the marginal indemnity

$$
\frac{\partial I(x)}{\partial x}=\frac{\operatorname{Ra}(A)}{R a(A)+\frac{C^{\prime \prime}(|I(x)|)}{1+C^{\prime}(|I(x)|) \cdot \operatorname{sgn}(I(x))}},
$$

with $R a(A)=-\frac{U^{\prime \prime}(A)}{U^{\prime}(A)}$ denoting absolute risk aversion. Consequently, in contrast to Gollier, $\frac{\partial I(x)}{\partial x} \leq 1$. Although marginal indemnity increases with insured's risk aversion, full marginal reimbursement becomes optimal only if risk aversion approaches infinity. The optimal indemnity schedule is illustrated inFig. 1:

\footnotetext{
2 The usual constraint $I(x) \leq x$ is disregarded for two reasons: First, it does not make much sense in a model which does not allow for informational asymmetries, specifically moral hazard. Second, it will turn out that this restriction is not binding anyway if costs are convex.

${ }^{3}$ As can be seen from (5), negative indemnities are restricted: The marginal costs they induce must be lower than 1 .
} 
Fig. 1 Optimal indemnity with increasing marginal costs

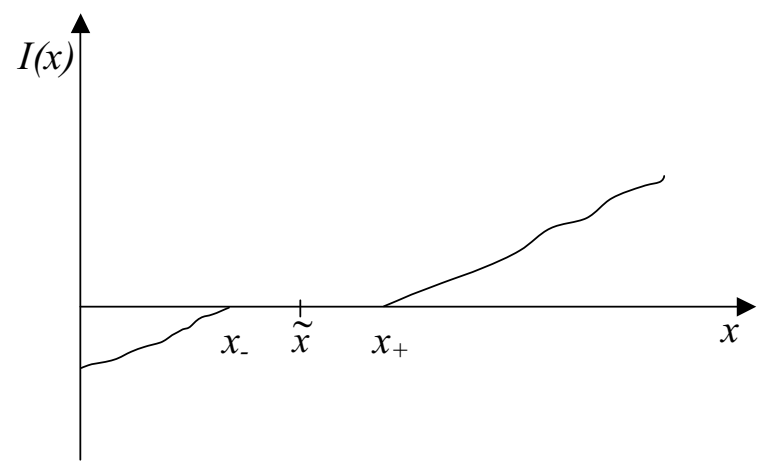

\section{How restrictive is the non-negativity constraint?}

Since $x_{-}$and $x_{+}$coincide for infinitely risk averse insured, we can define $\tilde{x}$ as the limit of $x_{-}$and $x_{+}$. However, it may turn out that $\tilde{x}$ is non-positive, in which case the nonnegativity constraint would not bind and usual insurance contracts of the deductible type would be efficient.

The optimal $\tilde{x}$ is the one that minimizes expected transaction costs $\left(\int_{0}^{L} C(|I(x-\tilde{x})|) f(x) d x\right)$. Individuals' risk aversion and marginal costs, on the other hand, determine the distance between $x_{-}$and $x_{+}$with $\tilde{x} \in\left(x_{-}, x_{+}\right)$as well as the slope of the indemnity function.

Differentiating expected transaction costs w.r.t. $\tilde{x}$ and setting the result to zero yields

$$
\int_{0}^{\tilde{x}} C^{\prime}(|I(x-\tilde{x})|) \cdot I^{\prime}(x-\tilde{x}) f(x) d x=\int_{\tilde{x}}^{L} C^{\prime}(|I(x-\tilde{x})|) \cdot I^{\prime}(x-\tilde{x}) f(x) d x .(8)
$$

Gollier (1987) shows that if marginal costs are constant, $\tilde{x}$ always coincides with the median of the loss distribution. Consequently, in this case the non-negativity constraint is never binding if the probability of a positive loss is lower than $1 / 2$.

However, with non-constant marginal costs, this result no longer holds. Suppose an asymmetric loss distribution with more mass on low losses, as it is characteristic for health insurance. If $\tilde{x}$ coincided with the median, high losses would deviate more from $\tilde{x}$ than low losses, causing higher absolute indemnities than low losses do. Higher indemnities go along with higher marginal costs. To prevent the RHS of (8) to exceed its LHS, one has to chose $\tilde{x}$ such that Eq. (8) is met again, while the distance between $x_{-}$and $x_{+}$is determined by Eq. (6) and depends on the insured's risk aversion and the marginal costs at $I(x)=0$. Consequently, for sufficiently risk averse insured and low marginal costs at $I(x)=0$, the interval between the two losses $x_{-}$and $x_{+}$which is compatible with both Eqs, (6) and (8) may not include the median but be on its RHS.

Consequently, for convex costs an imposed non-negativity constraint may be binding more often than Gollier suggests. Returning to the example from health insurance, the non-negativity constraint may also bind for insured who are not chronically ill. The effect of a non-negativity constraint to the insured is that they are urged to accept higher marginal costs to reduce the variance of their final wealth. ${ }^{4}$

\footnotetext{
${ }^{4}$ This effect becomes most obvious for insured with risk aversion approaching infinity, inducing full marginal indemnity. In order to stabilize their final income they can only reduce their final losses to zero and have to bear the high marginal costs of the high indemnity payments from the insurer.
} 


\section{Conclusion}

Keeler [1974] and Gollier [1987] were the first to show that non-negativity constraint on indemnities may be binding. By deriving the properties of an optimal insurance contract for a broader class of convex cost functions, this paper shows that the optimal insurance contract generally does not show full marginal indemnity any more. Furthermore, the non-negativity constraint might be binding more often than Gollier suggests. Although the model does not deal with moral hazard, the results are nonetheless robust if insured can invest in self-insurance (loss reduction). The optimal indemnity schedule derived here provides incentives to do so (Winter, 2000).

All in all, the indemnity schedule derived in this paper could help to transfer Keeler's and Gollier's ideas into the domain of practical applications.

Acknowledgments The author wishes to thank Christian Gollier, Emmett Keeler, Boris Krey, Yves Schneider, Catarina Goulão and Peter Zweifel for encouraging and helpful comments on earlier versions of this paper. Finally, comments by two anonymous referees helped to improve the paper substantially.

\section{References}

ARROW, K.J. [1971]: Essays in the Theory of Risk Bearing. North-Holland: Amsterdam.

DRÈZE, J.H. [1981]: "Inferring Risk Tolerance from Deductibles in Insurance Contracts," The Geneva Papers on Risk and Insurance, 20, 48-52.

GOLLIER, C. [1987]: "The Design of Optimal Insurance Contracts without the Nonnegativity Constraint on Claims," The Journal of Risk and Insurance, 54, 314-324.

GOLLIER, C. and SCHLESINGER, H. [1996]: "Arrow's Theorem on the Optimality of Deductibles: A Stochastic Dominance Approach,” Economic Theory, 7, 359-361.

GOULD, J.P. [1969]: "The Expected Utility Hypothesis and the Selection of Optimal Deductibles for a given Insurance Policy," The Journal of Business, 42, 143-151.

HUBERMAN, G., MAYERS, D., and Smith, C.W. Jr [1983]: “Optimal Insurance Policy Indemnity Schedules," The Bell Journal of Economics, 14, 415-426.

KEELER, E. [1974]: “A Note on Optimal Insurance,” The Rand Paper Series P-5173.

MOFFET, D. [1977]: “Optimal Deductible and Consumption Theory,” The Journal of Risk and Insurance, pp. 669-882.

MOSSIN, J. [1968]: “Aspects of Rational Insurance Purchasing,” Journal of Political Economy, 76, 553568.

RAVIV, A. [1979]: “The Design of an Optimal Insurance Policy,” The American Economic Review, 69, 84-96.

SCHLESINGER, H. [1981]: “The Optimal Level of Deductibility in Insurance Contracts," The Journal of Risk and Insurance, 465-481.

SPAETER, S. and ROGER, P. [1997]: "The Design of Optimal Insurance Contracts: A Topological Approach," The Geneva Papers on Risk and Insurance, 22, 5-19.

WINTER, R.A. [2000]: “Optimal Insurance under Moral Hazard,” Handbook of Insurance, in G. Dionne, (ed.): Boston, Kluwer Academic, Dordrecht and London, 155-183. 